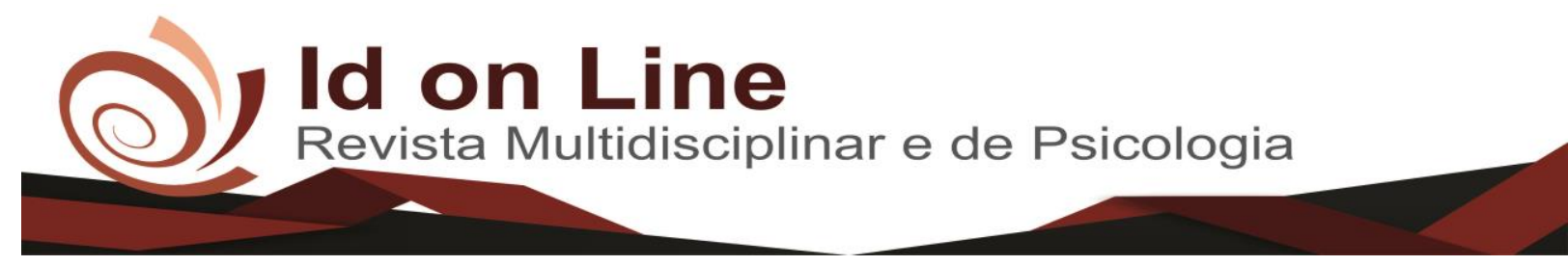

Artigo

\title{
Análise do Conhecimento e Atitudes dos Profissionais das USFs da Zona Urbana sobre Notificação de Reações Adversas
}

\author{
Alana Ferraz Flores ${ }^{l}$; Rodrigo Santos Damascena ${ }^{2}$
}

\begin{abstract}
Resumo: Os medicamentos são instrumentos indispensáveis no esquema terapêutico de diversas doenças, no qual, auxiliam na prevenção e progressão da mesma. As reações adversas aos medicamentos (RAM) são eventos imprevisíveis, de intensidade leve ou grave (como hospitalização, incapacidade e óbito) e que pode acometer qualquer pessoa quando administrar um medicamento em dose terapêutica. Objetivo: O presente estudo tem por objetivo analisar o conhecimento e atitudes dos profissionais das USFS da zona urbana sobre notificação de reações adversas. Materiais e Métodos: Trata-se de um estudo seccional descritivo conduzido no município de Vitória da Conquista por meio de um questionário estruturado e auto preenchido. A pesquisa envolveu 41 entrevistados de 3 categorias profissionais: 21 enfermeiros $(51,22 \%)$, 12 médicos $(29,27 \%)$ e 8 odontólogos $(19,51 \%)$. Resultados e discussão: Observou-se predominância do gênero feminino $(75,61 \%)$, bem como das faixas etárias de $36-40(26,83 \%)$ e $41-45$ anos $(21,95 \%)$. A maioria dos entrevistados tinham acima de seis anos de formação $(40 \%)$, a maioria $(80 \%)$ porta títulos de especialização. A compreensão quanto ao nível de conhecimento acerca de RAM, apenas $46,34 \%(n=19)$ dos profissionais o classificaram como razoável e $41,46 \%(\mathrm{n}=17)$ de forma satisfatória. Os profissionais de Saúde da Família que participaram do presente estudo caracterizaram o conhecimento limitado sobre as notificações de reações adversas, o que evidencia a necessidade de ações estratégicas (palestras; distribuição de informativos e folhetos; capacitações) voltadas para a educação continuada da equipe multiprofissional. Conclusão: De acordo com os dados obtidos nesse estudo, conclui-se que o incentivo à notificação de RAM é considerado elemento chave para o fortalecimento dos sistemas de monitorização, utilizado pela farmacovigilância, sendo de suma importância para a melhoria da qualidade e do uso racional dos medicamentos. Porém, enfatiza-se a necessidade de capacitação como estratégia de preparação do profissional para notificação espontânea.
\end{abstract}

Palavras-Chave: Conhecimento. Centros de Saúde. Área Urbana. Assistência Ambulatorial.

\section{Analysis of the Knowledge and Attitudes of the Professionals of USFs of the Urban Area on Notification of Adverse Reactions}

\begin{abstract}
Medications are indispensable tools in the therapeutic scheme of several diseases, in which they help in the prevention and progression of the same. Adverse Drug Reactions (ADRs) are unpredictable events of mild or severe severity (such as hospitalization, disability, and death) that can affect anyone when administering a medication at a therapeutic dose. Objective: The objective of this study is to analyze the knowledge and attitudes of USFS professionals in the urban area about the reporting of adverse reactions. Materials and Methods: This is a descriptive cross-sectional study conducted in the city of Vitória da Conquista through a structured and selfcompleted questionnaire. The survey involved 41 interviewees from 3 professional categories: 21 nurses $(51.22 \%)$, 12 physicians (29.27\%) and 8 dentists (19.51\%). Results and discussion: Female gender predominated $(75.61 \%)$, as well as age groups of 36-40 (26.83\%) and 41-45 years (21.95\%). Most respondents had over six years of training $(40 \%)$, most $(80 \%)$ holding specialization degrees. The understanding of the level of knowledge about ADR, only $46.34 \%(n=19)$ of the professionals classified it as reasonable and $41.46 \%(n=17)$ satisfactorily. The family
\end{abstract}

\footnotetext{
${ }^{1}$ Graduanda em Farmácia pela Faculdade Independente do Nordeste, FAINOR, Brasil. alanaferraz-f@ @otmail.com

${ }^{2}$ Mestrado Profissional em Saúde Pública pela Fundação Oswaldo Cruz, Brasil. Farmacêutico da Prefeitura Municipal de Vitória da Conquista-BA, Brasil. Professor orientador da Faculdade Independente do Nordeste, FAINOR.
} 
health professionals who participated in the present study characterized the limited knowledge about adverse reactions reports, which evidences the need for strategic actions (lectures, distribution of information and leaflets, training) aimed at the continuing education of the multiprofessional team. Conclusion: According to the data obtained in this study, it is concluded that the incentive to the notification of ADR is considered a key element for the strengthening of the monitoring systems, used by pharmacovigilance, being of paramount importance for the improvement of quality and rational use of medicines. However, the need for training as a strategy to prepare professionals for spontaneous reporting is emphasized.

Keywords: Knowledge. Health Centers. Urban Area. Ambulatory Care.

\section{Introdução}

Os medicamentos são instrumentos indispensáveis no esquema terapêutico de diversas doenças, no qual, auxiliam na prevenção e progressão da mesma. Entretanto, o uso irracional pode acarretar em prejuízos à saúde daqueles que os utilizam, como intoxicações e reações adversas. O Brasil é um dos países que mais consome medicamentos do mundo, ocupando o ranking de quarto lugar, em 2017 (SOARES; ARTICO; MENEZES, 2017).

As reações adversas aos medicamentos (RAM) são eventos imprevisíveis, de intensidade leve ou grave (como hospitalização, incapacidade e óbito) e que pode acometer qualquer pessoa quando administrar um medicamento em dose terapêutica (FONTELES; et al., 2009). Estudo realizado pela British Medical Association (2006) aponta que a mortalidade da população, devido à RAM equivale a 0,15\% (FERREIRA; et al., 2017).

Assim, quando um medicamento é disponibilizado para comercialização, é necessário que ele tenha passado por testes pré-clínicos e clínicos que comprovem a sua eficácia, eficiência e segurança. Entretanto, os testes clínicos não conseguem compreender um grande número de indivíduos e não detectam ocorrências de reações adversas graves, por isso, existe a farmacovigilância, que assegura um monitoramento e vigilância competente, capaz de identificar e prevenir qualquer evento que envolva o medicamento, facilitando a notificação no mercado farmacêutico e preservando a saúde da população (DRESCH, 2006).

As reações adversas podem ser notificadas através de três principais maneiras, notificação espontânea, estudos epidemiológicos (caso-controle e coorte) e técnicas epidemiológicas específicas (como por exemplo, sistemas de monitoramento em pacientes hospitalizados). A notificação é um ato que envolve, principalmente os profissionais de saúde, 
para uma possível descrição do efeito adverso nos sistemas de notificação e melhor conduta de orientação para o indivíduo acometido (DRESCH, 2006).

Os profissionais de saúde são fontes de informações sobre possíveis RAM, e alguns dos acidentes raros e/ou tardios só foram descobertas graças as observações de médicos que associaram o aparecimento de agravo/danos à saúde a uma origem medicamentosa. A responsabilidade de notificar engloba os profissionais de saúde que está em contato com o medicamento e paciente, gerenciando, dessa forma, a qualidade da assistência e segurança do paciente (FERREIRA et al., 2013).

Entretanto, acredita-se que muitas RAM ainda sejam subnotificadas. Em consenso com estudo proposto por Varallo e colaboradores. (2014) e Granas e colaboradores. (2007) apenas 5 a $10 \%$ dos eventos envolvendo medicamentos são registrados e uma das justificativas que influenciam esse dado é o pouco conhecimento que os profissionais têm para identificar e notificar uma reação. Este artigo teve como objetivo analisar o conhecimento e atitudes dos profissionais das USFs da zona urbana sobre notificação de reações adversas.

\section{Materiais e Métodos}

Trata-se de um estudo seccional descritivo conduzido no município de Vitória da Conquista por meio de um questionário estruturado e auto preenchido com base em instrumento adaptado do estudo de Barreto; Simões (2008) e Pinheiro; Pepe (2011), e, também, nas boas práticas de farmacovigilância para as américas, material desenvolvido pela organização mundial de saúde (OMS), para auxiliar profissionais de saúde a identificar e notificar problemas relacionados aos medicamentos e reações adversas.

O local escolhido para realização do estudo foi o município de Vitória da Conquista, Bahia. Essa área está situada no semiárido nordestino e é conhecida como a terceira maior cidade do estado da Bahia. De acordo com as estimativas de 2012 existem aproximadamente 315.884 habitantes na cidade. Além disso, a economia é regida principalmente pela agricultura, pecuária e serviços ofertados a educação e saúde (BISPO JÚNIOR; MARTINS, 2012).

No que diz respeito à saúde, o município é referência para mais de 70 cidades circunvizinhas, sendo que na gestão do Sistema Único de Saúde (SUS), Vitória da Conquista é sede da Macrorregião de Saúde do sudoeste da Bahia (BISPO JÚNIOR; MARTINS, 2012). Com base nos registros da Secretaria Municipal de Saúde para atenção primária, o município 
possui 32 unidades de Saúde da Família (USF), sendo 16 USFs na zona urbana, com 28 equipes de saúde da família (ESFs).

Dessa forma, a presente pesquisa ocorreu em 16 USF, e a população de estudo foram os profissionais de saúde que trabalham nas USF. As USF que integram o município são compostas, em sua grande maioria, pela equipe multidisciplinar de médicos, enfermeiros, agentes comunitários de saúde, funcionários de serviço geral e vigilante (REIS; e colaboradores., 2013).

Os critérios de inclusão no estudo contemplaram profissionais de saúde de três categorias - dentista, enfermeiro e médico -, eleitos por serem ativos na assistência a pacientes, com interface direta com o medicamento como a prescrição e a administração. Critérios de exclusão para: os que não foram encontrados ou que se recusaram a participar da pesquisa.

A coleta de dados ocorreu em local privativo, dentro da USF, para evitar distrações e manter o sigilo das informações ofertadas. O pesquisador aplicou um questionário diretamente ao profissional de saúde, composto por questões com informações pertinentes ao tema, na qual abordaram os aspectos pessoais do profissional, o conhecimento da farmacovigilância e o manejo para lidar com uma possível notificação.

O questionário possuía duas seções: a primeira com dados socioeconômicos (gênero, idade, etc), e a segunda com questões específicas com o objetivo de avaliar os conhecimentos e as condutas dos profissionais de saúde em relação às notificações de reações adversas a medicamentos e à farmacovigilância, respectivamente (MODESTO et al, 2016).

As variáveis utilizadas para a caracterização do perfil profissiográfico dos profissionais de saúde foram: categoria profissional, tempo de formação, tempo de atuação na instituição, trabalhar em outro local, carga horária semanal total de trabalho; e para o perfil demográfico, foram utilizados gênero e idade (MODESTO et al, 2016).

Para verificar o nível de conhecimento dos profissionais em relação às reações adversas a medicamentos e farmacovigilância, foram adotados os seguintes critérios: conhecimento acerca da reação adversa a medicamentos, condutas adotas em relação a RAM, conhecimento sobre a possibilidade de realizar notificação adversa pela internet, e os conhecimentos institucionais e dos profissionais acerca do que pode atuar frente as notificações de RAM (MODESTO et al, 2016).

Os dados foram tabulados em programas disponíveis Microsoft Excel® 2010 e Microsoft Word® 2010 e, posteriormente, confrontadas na literatura científica.

Este estudo recebeu a aprovação do Comitê de Ética da Faculdade Independente do Nordeste- FAINOR através do parecer $\mathrm{n}^{\circ} 2.960 .903$. Os indivíduos envolvidos na pesquisa 
assinaram o Termo de Consentimento Livre e Esclarecido (TCLE), que assegurou a integridade do trabalho e esclareceu quaisquer dúvidas acerca do trabalho abordado.

\section{Resultados e Discussão}

Do universo de 86 profissionais possíveis para participarem da pesquisa, 41 responderam ao questionário. Os demais, ou não quiseram participar ou não foram encontrados após 2 visitas à USF.

Sendo assim, a pesquisa envolveu 41 indivíduos que foram entrevistados, sendo estes de 3 categorias profissionais diferentes: 21 eram enfermeiros (51,22\%), 12 médicos $(29,27 \%)$ e 8 odontólogos (19,51\%). Pôde-se observar a predominância do gênero feminino 75,61\% $(n=31)$, bem como das faixas etárias de 36-40 (26,83\%) e 41-45 anos $(21,95 \%)$. Os entrevistados tinham acima de seis anos de formação $40 \%(n=16)$ e acima de quinze anos $30 \%$ $(n=12)$ e $80 \%$ (n=31) tinham os títulos de especialização (Tabela 1).

Tabela 1- Distribuição dos profissionais de saúde segundo características demográficas e funcionais nas USFs de Vitória da Conquista, Bahia. Brasil, 2018.

\begin{tabular}{lcc}
\hline Características & $\mathrm{n}$ & $\%$ \\
\hline Gênero & 10 & 24,39 \\
Masculino & 31 & 75,61 \\
Feminino & & \\
Faixa etária & 7 & 17,07 \\
$25-30$ & 9 & 21,95 \\
$31-35$ & 11 & 26,83 \\
$36-40$ & 9 & 21,95 \\
$41-45$ & 4 & 9,76 \\
$46-50$ & 1 & 2,44 \\
$\geq 71$ & & \\
Vínculo com a prefeitura & 31 & 75,61 \\
Concursado & 3 & 7,32 \\
Contratado & 7 & 17,97 \\
Programa mais médico & & \\
Categoria profissional & 21 & 51,22 \\
Enfermeiro & 12 & 29,27 \\
Médico & 8 & 19,51 \\
Odontólogo & & \\
Tempo de formação & 6 & 15 \\
$01-05$ & 16 & 40 \\
$06-10$ & 6 & 15 \\
$11-15$ & & \\
& & 6
\end{tabular}




\begin{tabular}{lcc} 
Pós-graduação & & \\
Especialização & 31 & 80 \\
Mestrado & 7 & 17,5 \\
Não possui & 3 & 7,5 \\
Carga horária semanal (em horas) & & \\
Até 20 & 2 & 4,88 \\
$21-40$ & 38 & 92,68 \\
$\geq 41$ & 1 & 2,44 \\
\hline
\end{tabular}

Fonte: Pesquisa dos autores, 2018.

Tabela 2- Nível de informação e comportamentos acerca de reações adversas a medicamentos (RAM) referida pelos profissionais das USFs de Vitória da Conquista, Bahia. Brasil, 2018

\begin{tabular}{|c|c|c|}
\hline Variável & $\mathrm{n}$ & $\%$ \\
\hline \multicolumn{3}{|l|}{ Conhecimento sobre RAM } \\
\hline Insuficiente & 3 & 7,32 \\
\hline Razoável & 19 & 46,34 \\
\hline Satisfatório & 17 & 41,46 \\
\hline Plenamente satisfatório & 2 & 4,88 \\
\hline \multicolumn{3}{|l|}{ Costumam perguntar sobre RAM aos pacientes } \\
\hline Sempre & 17 & 42,5 \\
\hline Frequentemente & 13 & 32,5 \\
\hline Ocasionalmente & 10 & 25 \\
\hline \multicolumn{3}{|l|}{ Relatos dos pacientes sobre sinais e sintomas } \\
\hline Sempre & 2 & 4,88 \\
\hline Frequentemente & 8 & 19,51 \\
\hline Ocasionalmente & 29 & 70,73 \\
\hline Nunca & 2 & 4,88 \\
\hline \multicolumn{3}{|l|}{ Fonte de atualização sobre o tema que costuma utilizar } \\
\hline Livros textos especializados & 21 & 52,5 \\
\hline Dicionário de especialidades farmacêuticas & 8 & 20 \\
\hline Revistas cientificas & 13 & 32,5 \\
\hline Palestras & 15 & 37,5 \\
\hline Cursos & 18 & 45 \\
\hline Congressos & 11 & 27,5 \\
\hline Outros & 14 & 35 \\
\hline Não utiliza nenhuma fonte & 2 & 5 \\
\hline \multicolumn{3}{|l|}{ Relatos dos pacientes sobre RAM na USF } \\
\hline Sim & 33 & 84,62 \\
\hline Não & 6 & 15,38 \\
\hline \multicolumn{3}{|l|}{ Conduta na suspeita de RAM } \\
\hline Suspende o medicamento & 31 & 77,5 \\
\hline Ajusta a dose & 15 & 37,5 \\
\hline Muda a terapêutica medicamentosa & 25 & 62,5 \\
\hline Mantém medicamento e não trata sinais e sintomas & 6 & 15 \\
\hline Comunica ao médico & 18 & 45 \\
\hline
\end{tabular}


Registra notificação em formulário para notificação espontânea

\begin{tabular}{|c|c|c|}
\hline \multicolumn{3}{|c|}{ Possibilidade de notificação de reação adversa pela internet } \\
\hline Sim & 7 & 17,07 \\
\hline Não & 34 & 82,93 \\
\hline \multicolumn{3}{|c|}{$\begin{array}{l}\text { Possui cadastro no Sistema de Notificação em Vigilância } \\
\text { Sanitária }\end{array}$} \\
\hline Sim & 0 & 0 \\
\hline Não & 41 & 100 \\
\hline \multicolumn{3}{|c|}{$\begin{array}{l}\text { Existe algum protocolo ou manual a ser seguido quando algum evento adverso } \\
\text { acontece }\end{array}$} \\
\hline $\operatorname{Sim}$ & $\begin{array}{l}9 \\
3\end{array}$ & 21,95 \\
\hline Não & 2 & 78,05 \\
\hline \multicolumn{3}{|c|}{ Já realizou alguma notificação de reação adversa a medicamento } \\
\hline $\operatorname{Sim}$ & 4 & 9,76 \\
\hline Não & 37 & 90,24 \\
\hline \multicolumn{3}{|c|}{ Profissionais que podem realizar notificação RAM } \\
\hline Médico(a) & 40 & 97,56 \\
\hline Enfermeiro(a) & 37 & 90,24 \\
\hline Nutricionista & 20 & 48,78 \\
\hline Farmacêutico(a) & 34 & 82,93 \\
\hline Profissional de Educação Física & 12 & 29,27 \\
\hline Psicólogo(a) & 18 & 43,9 \\
\hline Odontólogo(a) & 37 & 90,24 \\
\hline Fisioterapeuta & 16 & 39,02 \\
\hline \multicolumn{3}{|c|}{ Já recebeu alguma capacitação para realizar notificação de RAM } \\
\hline Sim & 4 & 9,76 \\
\hline Não & 37 & 90,24 \\
\hline \multicolumn{3}{|c|}{ Essa capacitação foi realizada pelo município } \\
\hline $\operatorname{Sim}$ & 4 & 26,67 \\
\hline Não & 11 & 73,33 \\
\hline \multicolumn{3}{|c|}{ Gostaria de receber alguma capacitação } \\
\hline Sim & 40 & 97,56 \\
\hline Não & 1 & 2,44 \\
\hline
\end{tabular}

Fonte: Pesquisa dos autores, 2018.

Em relação à compreensão quanto ao nível de conhecimento acerca de RAM, 46,34\% $(n=19)$ dos profissionais foram classificados como razoável e 41,46\% (n=17) de forma satisfatória. Os profissionais de Saúde da Família que participaram do presente estudo caracterizaram-se como obtendo o "conhecimento limitado" sobre as notificações de reações adversas, o que evidencia a necessidade de ações estratégicas relacionadas a educação em saúde 
(palestras; distribuição de informativos e folhetos; capacitações) voltadas para a educação continuada da equipe multiprofissional, no sentido de promover melhorias na qualidade e segurança do paciente, mantendo uma relação direta com o progresso da capacidade de rendimento para o trabalho e auxiliando na prevenção das reações adversas.

Dos profissionais entrevistados, 42,50\% $(\mathrm{n}=17)$ declararam o hábito de sempre perguntar a seus pacientes sobre o surgimento de sinais e sintomas relacionados com possíveis RAM, resultado semelhante ao encontrado no estudo de Salviano e colaboradores (2011), no qual a maioria dos profissionais referiram que sempre/frequentemente costumavam perguntar aos pacientes sobre os possíveis sinais e sintomas surgidos com o uso dos medicamentos, sendo que $70,73 \%$ ( $n=29)$ ocasionalmente relatam, durante as consultas, acerca de sinais e sintomas relacionados a reações que os mesmos atribuem ao uso de medicamentos, bem como sugerido em outros estudos (GONÇALVES, S.S;RODRIGUES, HMS;JESUS, I.S.;CARNEIRO, JAO;LEMOS, 2016; IURAS et al., 2016).

É fundamental que os profissionais informem aos pacientes sobre a possibilidade de RAM, haja vista que o surgimento dessas reações pode contribuir para a não-adesão ao tratamento farmacológico. Uma das principais razões, é a falta de informação sobre os medicamentos fazendo com que os indivíduos não cumpram adequadamente seus tratamentos (ARAÚJO et al., 2016; REMONDI; CABRERA; SOUZA, 2014; VIEIRA; LUCIA; ARRUDA, 2007).

A conduta e a orientação sobre a possibilidade de surgimento de RAM durante o tratamento farmacoterapêutico são aspectos importantes na informação ao usuário (DIAS; SOUZA; MISHIMA, 2016). A variedade de medicamentos e diferentes princípios ativos nas prescrições médicas pode contribuir negativamente na adesão ao tratamento farmacológico, tendo em vista o surgimento de interações, com isso, a possibilidade de RAM, no qual os pacientes acabam parando o tratamento sem orientação médica (FREITAS; NIELSON; PORTO, 2015; PENAFORTE et al., 2017).

A farmacovigilância é definida pela Organização Mundial de Saúde (WHO, 2002) como uma ciência e atividades, que têm a capacidade de detectar, prevenir, avaliar e compreender os efeitos adversos ou eventuais problemas relacionados aos medicamentos (PRM). Todavia, esse conceito não se concentra apenas em identificar reação adversa a medicamento (RAM), afim de evitar danos a população, mas, se expande para outras variáveis, como por exemplo, as plantas medicinais, vacinas e produtos biológicos (BARRETO; SIMÕES, 2008).

Em relação às fontes de atualização sobre RAM, a maioria dos enfermeiros, médicos e odontólogos referiram utilizar mais de uma fonte, sendo os livros e textos os mais citados $20 \%$ 
$(\mathrm{n}=21)$, cursos $17 \%(\mathrm{n}=18)$, palestras $17 \%(\mathrm{n}=15)$, revistas cientificas $13 \%(\mathrm{n}=13)$ e congressos $11 \%(n=11)$. Tais achados se encontram em consonância com os resultados do estudo de Pinheiro, Pepe (2011) no qual as fontes preferenciais utilizadas pelos profissionais eram livrostextos e revistas cientificas.

Outro dado importante demonstra que $85 \%(\mathrm{n}=33)$ dos profissionais informaram que os pacientes atendidos nas unidades de saúde relataram ter tido alguma reação adversa a medicamento. Entretanto, a maior parte dos profissionais $82,93 \%(n=34)$ mencionaram não ter conhecimento sobre notificação de reações adversas pela internet e 78,05\% (n=32) informaram que na unidade de saúde não existe nenhum protocolo ou manual a ser seguido, quando algum evento adverso acontece (RESENDE; SANTOS-, 2015).

Com a falta de um protocolo ou POP dificultam os profissionais de notificarem, devido à falta de prática e treinamento dos mesmos, evidenciando que mesmo aqueles que sabem o que é RAM, os profissionais de saúde desconhecem o escopo das atividades da farmacovigilância e preenchimento dos formulários de notificação. A importância da educação tem a finalidade de proporcionar conhecimento e ocasionar mudanças de paradigmas na atuação destes profissionais perante as notificações, para que assim assimilem que tais atividades fazem parte de suas responsabilidades.

Todos os profissionais referiram adotar alguma conduta na presença de suspeita de RAM. A maioria 92,5\% ( $\mathrm{n}=37)$ registram em prontuário, 77,5\% ( $\mathrm{n}=31)$ suspendem o medicamento, $72,5 \%(\mathrm{n}=29)$ orientam o paciente sobre reação adversa ao medicamento, $62,5 \%$ $(n=25)$ mudam a terapêutica medicamentosa e $45 \% \quad(n=18)$ comunicam ao médico. Os enfermeiros, diante de suspeitas de RAM, adotam a conduta de registrar no prontuário e comunicar o fato à categoria médica, essa conduta corrobora com os estudos de Modesto e colaboradores (2015) onde a maioria relatou que registram no prontuário.

A suspensão do medicamento, orientação ao paciente sobre RAM e mudança da terapêutica medicamentosa são condutas frequentemente adotadas pelos profissionais, convergindo com os achados de Salviano e colaboradores (2011) onde a mudança e suspensão dos medicamentos foram as condutas mais referidas diante da observação.

A maior parte dos entrevistados mencionou que todos os profissionais da área da Saúde podem notificar as suspeitas de RAM, sendo a indicação da categoria médica $(97,56 \%, n=40)$ foi quase unânime nessa resposta, seguido de enfermeiro e odontólogo $(90,24 \%, n=37)$ e farmacêutico $(82,93 \%, n=34)$. Nesse aspecto, um sistema de farmacovigilância não funciona sem a junção de seus notificadores. Consequentemente os médicos, odontólogos e enfermeiros das USFs precisam integrar-se para a implantação de um programa de notificação. Além disso, 
no presente estudo observou-se que a falta de atuação ativa e compreensão de que todos os profissionais de saúde podem notificar as suspeitas de reação adversa faz com que os mesmos deixem de notificar por desconhecimento do assunto.

Em relação a capacitação profissional, 90,24\% (n=37) dos participantes sinalizaram que nunca receberam alguma capacitação para realizar notificação e 97,56\% (n=40) relataram que gostariam de receber alguma capacitação, assim, é fundamental que tenha a implementação de um programa de notificação RAM nas unidades de saúde da família que está no desenvolvimento de treinamento e na participação ativa dos profissionais de saúde em realizar notificações suspeitas de RAM e outras experiências pertinentes, dessa forma cada um, na sua área, coopera para o planejamento de estratégias para promover a promoção de uma vigilância ativa da segurança dos medicamentos (FREIRE et al., 2015; SEBASTIÃO et al., 2016).

O comprometimento da adesão e ação dos medicamentos ao tratamento, e sua interação entres os fármacos, está ligada ao uso irracional dos medicamentos podendo contribuir para o surgimento de RAM. Essa problemática está presente no setor Saúde e traz consigo diversas complicações (LIMA et al., 2016).

\section{Considerações Finais}

De acordo com os dados obtidos nesse estudo, conclui-se que o incentivo à notificação de RAM é considerado elemento chave para o fortalecimento dos sistemas de monitorização, utilizado pela farmacovigilância, sendo de suma importância para a melhoria da qualidade e do uso racional dos medicamentos. Porém, enfatiza-se a necessidade de capacitação como estratégia de preparação do profissional para notificação espontânea. A notificação de RAM é vista como uma ferramenta regulatória fundamental para alimentar o sistema de farmacovigilância no país. Quanto mais forte for o sistema nacional de farmacovigilância e de notificações de RAMs, mais provável será que decisões regulatórias equilibradas sejam tomadas pelos órgãos regulatórios como a Agência Nacional de Vigilância Sanitária (ANVISA) no Brasil. 


\section{Referências}

ADAMI, E., R.; CHEMIN., M., R., C.; FRANÇA, B., H., S. Aspectos éticos e bioéticos da pesquisa clínica no Brasil. Estudos de Biologia, v. 36, 2014.

ARAÚJO, M. da C. F. et al. Perfil de Não Adesão ao Tratamento de Usuários com Diabetes e Hipertensão em uma Unidade de Saúde da Família. Ensaio e Ciência: Biológicas, Agrárias e da Saúde, [s.1.], v. 20, p. 43-48, 2016.

BARRETO, G., G; SIMÕES, M., J., S. Fatores relacionados à adesão das drogarias e farmácias de Tatuí-SP no projeto "Farmácias Notificadoras". Revista de Ciências Farmacêuticas Básica e Aplicada, v. 29, n.1, p. 59-68, 2008.

BERTOLDI, A., D.; DAL PIZZOL, T., DA S.; RAMOS, L., R.; MENGUE, S., S.; LUIZA, V., L.; TAVARES, N., U., L.; FARIAS, M., R.; OLIVEIRA, M., A.; ARRAIS, P., S., D. Perfil sociodemográfico dos usuários de medicamentos no Brasil: resultados da PNAUM 2014. Revista de Saúde Pública, v. 50, s. 2, 2016.

BOTH, J., S.; KAUFFMANN, C.; ELY, L., S.; DALL'AGNOL, R.; RIGO, M., P., M.; TEIXEIRA, M., F., N.; DE CASTRO, L., C. Cuidado farmacêutico domiciliar ao idoso: análise de perfil e necessidades de promoção e educação em saúde. Caderno Pedagógico, v. 12, n. 3, 2015.

DIAS, E. G.; SOUZA, E. L. S.; MISHIMA, S. M. Contribuições da enfermagem na adesão ao tratamento da hipertensão arterial : uma revisão integrativa da literatura brasileira. Revista de Epidemiologia e Controle de Infecção, [s.1.], v. 6, n 3, p. 138-44, 2016. ISSN: 22383360, DOI: 10.17058/reci.v6i3.7470.

DRESCH, C. A Farmacovigilância e a atenção primária à saúde: diálogo possível e necessário. Revista APS, v.9, n.1, p. 73-82, 2006.

FERREIRA, A., L.; ROCHA, C., P.; VIEIRA, L., M.; SANTÁNA DUSSE, L., M.; JUNQUEIRA, D., R., G.; CARVALHO, M., DAS G. Alterações hematológicas induzidas por medicamentos convencionais e alternativos. Revista Brasileira de Farmácia, v. 94, n. 2, p. 94-101, 2013.

FONTELES, M., M., DE F.; FRANCELINO, E., V.; SANTOS, L., K., X., DOS; SILVA, K., M.; SIQUEIRA, R.; VIANA, G., S., DE B.; VASCONCELOS, S., M., M., DE; SOUSA, F., C., F., DE; MONTEIRO, M., P. Reações adversas causadas por fármacos que atuam no sistema nervoso: análise de registros de um centro de farmacovigilância do Brasil. Revista de Psiquiatria Clínica, v. 36, n. 4, p. 137-44, 2009.

FREIRE, N. C. B. et al. REAÇÕES ADVERSAS RELACIONADAS À INFUSÃO DE CÉLULAS TRONCO HEMATOPOÉTICAS. Revista de Enfermagem UFPE on line, [s.l.], v. 9, p. 391-398, 2015. DOI: 10.5205/reuol.5221-43270-1-RV.0901supl201518.

FREITAS, J. G. A.; NIELSON, S. E. D. O.; PORTO, C. C. Adesão ao tratamento farmacológico em idosos hipertensos: uma revisão integrativa da literatura. Revista da Sociedade Brasileira de Clínica Medica, [s.1.], v. 13, nº 1, p. 75-84, 2015. ISSN: 1679-1010. 
GONÇALVES, M., J., C.; AZEVEDO JÚNIOR, S., A.; DA SILVA, J.; SOUZA, L., DO N. A importância da assistência do enfermeiro ao idoso institucionalizado em instituição de longa permanência. Revista Científica de Enfermagem, v. 5, n. 14, 2015.

GONÇALVES， S.S;RODRIGUES， HMS;JESUS， I.S.;CARNEIRO, JAO;LEMOS， G. Ocorrencia Clinica De Interações Medicamentosas Em Prescrições De Pacientes Com Suspeita De Reação Adversa Internados Em Um Hospital No Interior Da Bahia. Rev.Aten.Saúde,São Caetanodo Sul, [s.1.], v. 14, nº 48, p. 32-33 ABR./JUN.29016, 2016. DOI: 10.13037/rbcs.vol14n48.3088.

GRANAS, A., G.; BUAJORDET, M.; STERBERG-NILSEN, H.; HARG, P.; HORN, A., M. Pharmacists' attitudes towards the reporting of suspected adverse drug reactions in Norway. Pharmacoepidemiol. Drug Saf., v. 16, n. 4, p. 429-34, 2007

HERDEIRO, M., T.; FERREIRA, M.; RIBEIRO-VAZ, I.; JUNQUEIRA POLÓNIA, J.; COSTA-PEREIRA, A. O Sistema Português de Farmacovigilância. Acta Médica Portuguesa, v. 25, n. 4, p. 241-249, 2012.

IURAS, A. et al. Prevalência da automedicação entre estudantes da Universidade do Estado do Amazonas (Brasil). Revista Portuguesa de Estomatologia, Medicina Dentaria e Cirurgia Maxilofacial, [s.1.], v. 57, $\mathrm{n}^{\mathrm{o}}$ 2, p. 104-111, 2016. ISSN: 16476700, DOI: 10.1016/j.rpemd.2016.01.001.

LIMA, T. A. M. De et al. Análise de potenciais interações medicamentosas e reações adversas a anti-inflamatórios não esteroides em idosos. Rev. Bras. Geriatria e Gerontologia, [s.1.], v. 19, no 3, p. 533-544, 2016.

MACEDO, G., R.; DO CARMO, B., B.; DE CASTRO, G., F., P.; CORREA, J., B. O poder do marketing no consumo excessivo de medicamentos no Brasil. Revista Transformar, 2016.

MASTROIANNI, P., C.; VARALlO, F., R.; BARG, M., S.; NOTO, N., A.; GALDURÓZ, J., C., F. Contribuição do uso de medicamentos para internação hospitalar. Revista Brasileira de Ciências Farmacêuticas, v. 45, p. 163-170, 2009.

MEDEIROS, J., C.; FERREIRA, S., C.; PAIXÃO, M., N., DE J. Conhecimento sobre reação adversa a medicamentos entre graduandos de farmácia e enfermagem. Revista de Pesquisa em Saúde, v. 18, n. 1, p. 13-17, 2017.

NUNES, C., R., DE M.; ALENCAR, G., DE O.; BEZERRA, C., A.; BARRETO, M., DE F., R.; SARAIVA, E., M., S. Panoramas das intoxicações por medicamentos no Brasil. Revista eciência, v. 5, n. 2, p. 98-103, 2017.

PENAFORTE, K. L. et al. Association between polypharmacy and the adherence to pharmacological treatment in patients with diabetes. Revista da Rede de Enfermagem do Nordeste, [s.1.], v. 18, $\mathrm{n}^{\mathrm{o}}$ 5, p. 631-638, 2017. ISSN: 21756783, DOI: 10.15253/21756783.2017000500010. 
PINHEIRO, H., C., G.; PEPE, V., L., E. Reações adversas a medicamentos: conhecimento e atitudes dos profissionais de saúde em um hospital-sentinela de ensino do Ceará-Brasil, 2008. Epidemiologia e Serviços de Saúde, v. 20, n. 1, 2011.

REMONDI, F. A.; CABRERA, M. A. S.; SOUZA, R. K. T. De. [Non-adherence to continuous treatment and associated factors: prevalence and determinants in adults 40 years and older]. Cad Saúde Pública, [s.1.], v. 30, nº 1, p. 126-36, 2014. ISBN: 0102-311X, ISSN: 0102-311X, DOI: 10.1590/0102-311X00092613.

RESENDE, L.; SANTOS-. Fatores de risco associados às reações adversas a medicamentos antituberculose. ET J Bras Pneumol, [s.1.], v. 41, n 1, p. 77-89, 2015. ISSN: 18063756, DOI: 10.1590/S1806-37132015000100010.

SALES, C., C., F.; MESCHIAL, W., C.; OLIVEIRA, M., L., F., DE. Construção de oficinas pedagógicas para prevenção das intoxicações infantis. Arquivos de Ciências da Saúde da UNIPAR, v. 22, n. 1, 2018.

SEBASTIÃO, M. et al. Incidência de Reações Adversas a Medicamentos nos Serviços de Medicina e Terapia do Hospital Central Josina Machel , em 2014 Incidence of Adverse Reactions to Medicinal Products in Josina Machel Central Hospital during the Year of 2014. Revista Portuguesa de Farmacoterapia, [s.1.], v. 8, p. 105-111, 2016.

SILVA, N., R., DA; ABJAUDE, S., A., R.; MARQUES, L., A., M., RASCADO, R., R. Centro de Farmacovigilância da Unifal (CEFAL): promoção da segurança, efetividade e qualidade do medicamento. Cidadania em Ação: Revista de Extensão e Cultura, v.7, n. 1, 2013.

VIEIRA, M. A.; LUCIA, A.; ARRUDA, G. De. Produção bibliográfica sobre adesão/nãoadesão de pessoas ao tratamento de saúde. REVISÃO REVIEW, [s.1.], p. 2299-2306, 2007. ISSN: 1413-8123, DOI: 10.1590/S1413-81232008000900034.

Como citar este artigo (Formato ABNT):

FLORES, Alana Ferraz; DAMASCENA, Rodrigo Santos. Análise do Conhecimento e Atitudes dos Profissionais das USFs da Zona Urbana sobre Notificação de Reações Adversas . Id on Line Rev.Mult. Psic., 2019, vol.13, n.43, p. 614-626. ISSN: 1981-1179.

Recebido: 22/11/2018;

Aceito: $23 / 11 / 2018$ 